Cosche, Cosa nostra : les structures organisationnelles de la criminalité mafieuse en Sicile

\title{
Raimondo Catanzaro
}

\section{(2) OpenEdition Journals}

Édition électronique

URL : http://journals.openedition.org/conflits/105

DOI : 10.4000/conflits.105

ISSN : $1777-5345$

Éditeur :

CCLS - Centre d'études sur les conflits lilberté et sécurité, L'Harmattan

Édition imprimée

Date de publication : 17 octobre 1991

ISSN : 1157-996X

Référence électronique

Raimondo Catanzaro, « Cosche, Cosa nostra : les structures organisationnelles de la criminalité mafieuse en Sicile », Cultures \& Conflits [En ligne], 03 | automne 1991, mis en ligne le 31 décembre 2002, consulté le 30 mars 2021. URL : http://journals.openedition.org/conflits/105 ; DOI : https:// doi.org/10.4000/conflits. 105

Ce document a été généré automatiquement le 30 mars 2021

Creative Commons License 


\title{
Cosche, Cosa nostra : les structures organisationnelles de la criminalité mafieuse en Sicile
}

\author{
Raimondo Catanzaro
}

1 Dans l'analyse de la structure organisationnelle de la criminalité mafieuse sicilienne, deux problématiques se sont toujours opposées. Nous pouvons commodément les schématiser ainsi : d'un côté nous avons la thèse de l'existence d'une organisation unique et coordonnée, de l'autre celle d'une pluralité d'organisations dispersées sur le territoire. Ces deux thèses ont des conséquences différentes sur la nature et la définition de la criminalité mafieuse. De plus, elles débouchent sur des stratégies différentes quant aux formes de lutte à envisager contre les mafieux. Comment les départager?

2 L'objet de cet article est d'analyser les conditions qui peuvent déterminer empiriquement la prééminence d'une thèse sur l'autre dans des contextes sociohistoriques déterminés ; cela nous amènera à déplacer les enjeux du débat, focalisés en Italie, depuis longtemps sur la structure organisationnelle, pour mieux s'entendre sur ce que peut être une criminalité de type mafieux.

Les cellules mafieuses comme structures informelles, fluides, à fin pratique (task oriented)

3 Dans l'essai fameux sur la mafia, écrit après l'homicide de Notarbartolo et ses suites judiciaires, Gaetano Mosca écrivait qu'il n'existe pas "une quelconque organisation qui réunit et dirige en un seul faisceau toutes les forces de la mafia"(1).

4 Cette idée d'une mafia constituée par une multiplicité d'organisations, aux liens relâchés, trouvait son origine dans les analyses faites par les préfets et magistrats de Sicile qui furent confrontés au problème dès les années 18 ?0-1880. Par exemple, le prêteur de Ravanusa (Agrigente) présentait la mafia comme "une vaste réunion de personnes de toutes classes, de toutes sphères sociales, sans liens apparents, dans le but de pourvoir aux intérêts communs, quelqu'ils soient"(2). 
5 En 1874, le préfet de Trapani fondait la distinction entre la camorra et la mafia sur l'élément organisationnel : "Les Camorristes forment une secte, ont des règles propres, ont leur propre hiérarchie, ont un langage astucieux, ont aussi un simulacre de jurés. Les mafieux, au contraire n'ont ni organisation ni normes régulatrices"(3).

6 La difficulté de repérer le type particulier d'organisation de la criminalité mafieuse s'explique par la combinaison d'activités licites et illicites qui la caractérise. C'est un fait que les mafieux furent traditionnellement des hommes d'honneur, révérés dans leur communauté d'appartenance. D'où la difficulté de percevoir la continuité de la structure organisationnelle mafieuse. Son caractère instable, ou mieux, fluide, a malgré tout été bien compris par le préfet Rasponi, qui dans un rapport de Palerme au ministère de l'Intérieur en 1875 rendait compte que "les mêmes malandrins ou malfaiteurs... n'ont pas une véritable association permanente, avec des liens et des pactes reconnus. Mais ils se connaissent mutuellement, s'habillent selon une mode uniformisée, et s'organisent selon les mêmes modalités d'action. Au besoin ils se réunissent, se concertent et s'entraident pour réussir leurs opérations. Après avoir commis un délit ou réalisé une action mafieuse, ils se partagent le butin puis se séparent et font mine de ne plus se connaître, sauf lors d'une autre occasion où ils se réunissent à nouveau"(4).

7 Les caractères de fluidité, de fin pratique, sont bien synthétisés, dans la définition donnée par Jane et Peter Schneider(5) concernant l'organisation mafieuse. Selon ce modèle, les fonctions d'organisation et de direction sont toutes attribuées au chef du groupe mafieux. La division du travail à l'intérieur est rudimentaire. Leopoldo Franchetti décrit avec beaucoup d'efficacité cette situation à propos du chef mafieux : "Celui-ci tient dans cette industrie délictueuse le rôle du capitaliste, de l'impresario et du directeur. Il est à la base de cette unité qui donne à la mafia son apparence de force inéluctable et implacable ; il règle la division du travail et des fonctions, la discipline entre les ouvriers de cette industrie... Il lui revient de juger selon les circonstances s'il convient de suspendre pendant un moment les violences, ou au contraire de les multiplier et de leur donner un caractère plus féroce. Il s'adapte aux conditions du marché pour choisir les opérations à effectuer, les personnes à exploiter, la forme des violences à utiliser. C'est son génie propre, qui lui permet de distinguer le moment où il convient de faire disparaître la personne récalcitrante aux ordres de la mafia, ou bien celui de lui faire accepter les accords avec un camouflet (abattage d'animaux ou destruction de matériels) ou même tout simplement de s'en tenir à un coup de semonce. Un ramassis d'individus ou une association d'assassins vulgaires de la basse classe de la société ne serait pas capable d'accomp1ir de telles choses délicates et recourrait toujours à la violence la plus brutale"(6). D'après ce modèle, on a une dispersion entre les diverses organisations mais une très forte centralisation à l'intérieur de chacune d'entre elles. Les relations passent toutes par le chef mafieux. Ses relations avec les autres membres composant la Cosca sont les suivantes : direction, choix des méthodes et des formes d'actions... Le terme traditionnel du langage argotique mafieux, "cosca", permet de saisir la structure puisqu'il désigne simultanément les feuilles d'artichauts et le groupe mafieux. Le terme "cacocciula", c'est-à-dire artichaut, désigne quant à lui le chef mafieux. Il est à noter l'étrange inversion des termes correspondant parfaitement du reste à la nature de l'organisation $\mathrm{du}$ groupe mafieux, qui tire sa force du chef mafieux puisque le complexe de l'organisation est désigné par un terme qui se réfère à un unique élément (la feuille de 
l'artichaut) tandis que le terme qui désigne l'ensemble, la "cacocciula", c'est-à-dire l'artichaut, désigne la figure de l'individu unique qui est le chef de l'organisation.

8 Ce modèle qui, à une époque récente, a été formulé théoriquement par des spécialistes comme Hess, Blok et les Schneider, permet de comprendre certaines fonctions des mafieux comme celle d'être un power broker (intermédiaire-courtier). Cette fonction de médiateur, telle qu'elle a été décrite par la littérature anthropologique, se caractérise par le fait qu'un individu met en relation des sujets qui se trouvent à des niveaux différents et qui n'ont pas de liaisons ou de relations directes en dehors de lui. Les médiateurs se placent sur les jointures critiques de ces relations, et traitent les tensions et les conflits qui se créent entre les différents groupes. Leur fonction ne consiste pas à éliminer les racines du conflit, mais plutôt à les maintenir en vie, de façon à garantir la persistance de ces tensions qui constituent leur raison d'être. Le pouvoir des médiateurs dérive donc de leur capacité d'agir dans toutes ces sphères(7).

De ce rôle joué par les brokers découlent d'autres exigences fonctionnelles. En effet, le broker exploite l'absence de liaisons ou de communications entre les sujets pour pouvoir affirmer son pouvoir. La structure de la "cosca" correspond parfaitement à cette exigence; la centralisation trouve à sa fondation une série de rapports "dyadiques" entre le chef mafieux et ses adhérents particuliers. La "cosca" est en effet organisée comme une sorte de confédération de dyades ou plus exactement car le terme fait penser à des relations deux à deux isolées, à des dyades reliées en étoile puisqu'un des éléments de toutes les dyades, le chef de la "cosca", est commun. De cette façon, il contrôle le flux des communications et empêche les communications directes entre les membres de la "cosca"; il peut également jouer le rôle de médiateur à leur égard, et exercer ainsi efficacement le contrôle interne de l'organisation. Ainsi la cosca, tout en étant fortement centralisée, conserve un caractère fluide et instable, sa création ou sa dissolution dépendant de la volonté exclusive du chef. Finalement, dans ce modèle, les "cosche" ou organisations mafieuses arrivent donc à être définies, simultanément, comme "un centre idéal d'intérêts communs" et un ensemble de "relations informelles liées entre elles"(8).

"Cosa nostra" comme organisation criminelle organisée

10 A l'inverse de cette thèse sur la fluidité organisationnelle, on en trouve une autre qui accentue au contraire les caractères d'organisation centralisée, secrète et structurée d'après des rituels précis. Cette thèse, qui a aussi des antécédents dans la littérature sur la mafia du siècle dernier et de la première moitié de celui-ci, a été mise à l'honneur dernièrement dans la presse à la suite des révélations des deux grands repentis, Buscetta et Contorno. Ces révélations qui reproduisent en grande partie, à propos de l'organisation criminelle, celles données en son temps par Joe Valachi sur la "cosa nostra" américaine, semblent remettre en cause les connaissances sur la structure organisationnelle mafieuse qui avaient été formulées par les anthropologues de la thèse sur la fluidité de la "cosca" et qui étaient considérées comme acquises(9).

11 En effet, sur la base des témoignages de Buscetta et Contorno, les juges palermitains sont arrivés aux conclusions suivantes : "La cosa nostra est organisée sur des structures hiérarchiques avec un sommet et un épicentre à Palerme, siège de l'organisme directorial de l'association dénommé "coupole" ou "commission". Contrairement à une conviction diffuse, la mafia de l'île n'est pas une structure formée d'associations indépendantes et contrastées, mais une organisation qui, même articulée et complexe, a une unité substantielle"(10). 

de structure organisationnelle : cosa nostra. La famille constitue la cellule de base de l'organisation. Il s'agit d'une structure à base territoriale qui contrôle un quartier ou la totalité d'une cité. de dix sous l'autorité d'un chef de dizaine (capodecina)". Les membres de la famille élisent le chef de famille. Celui-ci est assisté par un "conseiller", d'ordinaire une personne âgée ou du moins tenue en haute considération pour sa sagesse, et est aidé par un ou plusieurs vice-chefs choisis par le chef.

14 Trois familles ou plus, territorialement contiguës, constituent un "canton" et nomment un chef de "canton" qui peut être un chef de famille ou une personne différente.

Les chefs de canton constituent l'organisation collégiale dénommée "commission" ou "coupole" qui a une sphère d'action provinciale et dont la mission est d'assurer le respect des règles de la "cosa nostra" ainsi que de régler les différends entre les familles. La commission est présidée par un des chefs de canton dénommé secrétaire ou chef.

16 Il existe aussi, mais sur ce point les informations ne sont pas concordantes, un organisme supérieur de liaison, dénommé "inter provincial" à caractère secret et mystérieux et sur lequel Buscetta lui-même ne pouvait en dire davantage(12).

Dans cette description, on peut noter l'accent porté aux caractères de stabilité et d'unité de l'organisation mafieuse; caractères qui sont très marqués dans les révélations ultérieures concernant les procédures et les rituels d'affiliation des familles mafieuses. En effet, toujours d'après ces dépositions, les traditions exigent qu'avant toute admission d'un nouvel adepte dans une famille, les autres chefs de famille soient informés. Ainsi, la commission assure non seulement les fonctions de coordination, supervision et règlement des conflits entre les diverses familles, mais le degré d'autonomie de celles-ci se trouve fortement limité en ce sens qu'il n'y a pas de possibilité de recrutement autonome(13).

Le recrutement se fait par un rituel d'affiliation qui consiste à prêter serment de fidélité à la "cosa nostra". En présence de trois membres influents de la famille (d'après la version de Buscetta) ou de la famille entière (d'après la version de Contorno), le néophyte prend entre les mains l'image d'un Saint, la mouille avec le sang qui coule d'une piqûre d'aiguille, brûle l'image en continuant de la tenir dans la main et termine le serment par cette phrase : "ma chair doit brûler comme cette image si je ne demeure pas fidèle à mon serment" 14 .

19 La présence de ces rites indique donc que l'organisation mafieuse est une société secrète avec des règles impératives et très formalistes puisque les rites sont communs à toutes les familles. Seulement, quel crédit apporter à ces dépositions sinon le fait que la formule du serment rapportée par les repentis, démontre qu'ils ont une connaissance approfondie de la littérature criminologique positiviste? Nous devons trancher entre les deux thèses sans a priori. Le contenu de ces dépositions et les conclusions, qu'en tirent les juges palermitains, sont tout à fait en contradiction avec la thèse anthropologique présentant l'organisation mafieuse comme ayant un caractère fluide et non permanent où les soi-disant associations criminelles ne seraient en fait que des réseaux hiérarchisés de relations sociales. 
En premier lieu, la diffusion de la mafia en milieu urbain soumis à des processus profonds de différenciation sociale; processus qui ont comme conséquence de redessiner sans arrêt les délimitations territoriales et les sphères d'influences réciproques. En second lieu les nouvelles possibilités de "faire du fric" guident les acteurs mafieux émergeants et les poussent à défier les règles consolidées par la tradition et par les chefs de familles dominantes. Ces nouvelles conditions ont donc pour conséquence de provoquer un affaiblissement du chef de famille, à tel point que les familles rivales peuvent jouer sur ces divisions internes pour s'approprier des sections de territoire ou des sphères d'affaires appartenant à la famille en crise. L'alternance entre des phases de concurrence acharnée, de violence entraînant une augmentation des homicides et des règlements de compte, et des phases de paix mafieuse relativement stable, est en effet une constante dans l'histoire de la mafia. Traditionnellement, dans les phases de conflit aigu, on avait recours au chef de famille le plus qualifié pour trancher les controverses. Mais cela n'était possible que lorsque l'espace territorial où s'exerçaient des activités et s'exprimaient des intérêts mafieux était suffisamment délimité, lorsqu'il coïncidait avec le quartier ou avec le territoire de la commune. Le développement sur grande échelle des activités mafieuses a provoqué 
une crise de ces délimitations traditionnelles territoriales. Tandis que les familles continuaient à maintenir une base sociale d'enracinement territorial, le type des trafics dans lequel elles se sont engagées (en particulier celui des stupéfiants) a comme caractéristique de ne plus se dérouler sur des marchés locaux, les contraignant à tenir compte dans des situations répétées et constantes de dépassement des limites territoriales. Cette dynamique peut expliquer le changement d'organisation qu'on rencontre dans la mafia, et la nécessité absolue de coordonner les familles.

D'après le témoignage de $\mathrm{J}$. Bonanno aux Etats-Unis, cette nécessité apparaît dans les années trente, et la première réunion de la "commission" eut lieu à New York en 1931.

Mais la commission "ne faisait pas partie intégrante de la tradition. Il n'existait pas d'organisme de ce type en Sicile. La commission naquit de la nécessité d'une adaptation à la réalité américaine"(16). A Palerme, la même nécessité se manifeste lorsque la spéculation immobilière sur les terrains, la contrebande des tabacs, le début du trafic des stupéfiants, et le transfert en ville des mafieux (plutôt actifs jusqu'alors dans les villes de l'intérieur) fit monter brusquement le niveau des conflits entre les cosche qui se trouvaient en position de rivalité pour le contrôle des nouvelles possibilités d'enrichissement.

Des modifications ont donc eu lieu, mais on commettrait à l'inverse une erreur grossière si l'on considérait qu'elles ont radicalement changé les structures organisationnelles de la mafia, la transformant en une sorte d'organisation unitaire, centralisée et formellement structurée comme une organisation bureaucratique. En fait, les analogies, qui d'après les témoignages des mafieux paraissent associer les règles de fonctionnement de la mafia aux procédures d'associations régulières sont finalement très superficielles.

Commençons par la procédure "électorale" qui concerne le chef de famille. On serait fautif si l'on voulait considérer cette procédure comme un véritable vote, ainsi que cela existe dans une association ou un système démocratique. En effet le but du "vote" n'est pas de décider qui, parmi plusieurs candidats, va assumer le commandement. Il serait peu sensé par exemple de nommer chef de famille un candidat qui aurait obtenu la moitié des votes plus un contre un candidat qui en aurait eu la moitié moins un. Le but de ce que Buscetta appelle une "élection", mais qu'il faudrait pour être exact appeler "pronunciamento" (proclamation), vise à l'affirmation symbolique de l'unité de la famille à travers l'indication du consentement effectif de ses membres. C'est là une fonction sociale importante parce qu'à l'intérieur des familles mafieuses, la direction est assurée par le consentement et c'est seulement, dans des cas très spéciaux, qu'on recourt à l'usage de la force.

De la même manière, les rapports entre familles ne peuvent guère être éclairés par l'emploi d'une analogie avec les bureaux et systèmes organisés d'une entreprise dont le sommet directorial serait constitué par la "coupole". A la limite il y a plus d'analogie avec le système interétatique des relations internationales. Il s'agit en effet d'un véritable système de souverainetés concurrentes comme le démontrent plusieurs indicateurs, dont le plus caractéristique est constitué par la pratique répandue d'insérer ses hommes avec des fonctions d'espions, à l'intérieur des familles concurrentes. Et comme dans tous les cas de rapports entre souverains concurrents, l'organisme qui sert à régler la concurrence, à établir les normes, à éviter les conflits destructeurs, naît, meurt et ressuscite en fonction de l'évolution des rapports de force entre les familles mafieuses. L'évolution de la coupole sera elle-même fonction du 
caractère plus ou moins conflictuel de la concurrence interne et des relations entretenues avec les organes de l'État(17).

De même que les États recourent à la guerre pour régler les controverses quand les organismes internationaux de médiation ne réussissent plus à éviter les conflits, de même les familles mafieuses recourent aux "règlements de comptes" lorsqu'il n'y a plus de place pour la médiation. Dans ces situations, les organismes de coordination ne sont donc plus utilisés, ils perdent leurs fonctions et disparaissent de la scène. Ainsi, comme le montrent les enquêtes des juges palermitains, la "coupole" de la cosa nostra s'est créée puis, pendant la guerre des familles, a disparu un certain temps, avant de se reconstituer au milieu des années soixante-dix.

Dès lors ces considérations confirment que la "coupole" n'est pas la structure organisée centrale d'une association unique, mais une solution (avec un certain degré de formalisation des procédures) qui sert à régler le système sans cesse en mutation des conflits et alliances entre les familles. C'est donc les familles qu'il convient de regarder pour comprendre les mécanismes élémentaires de fonctionnement du système mafieux.

Violence et marché : les deux visages de la criminalité mafieuse

32 La confusion dans l'interprétation qui est à la base des opinions concernant la mafia vient donc de l'absence de distinction analytique entre les différents types d'activités que traitent les familles mafieuses. Cette distinction des deux types d'activité : violence et marché, dès qu'elle est faite, permet de mieux comprendre la véritable spécificité de la criminalité organisée mafieuse.

Les cosche mafieuses seront alors définies, en suivant Max Weber, comme "des groupements politiques dont le financement est intermittent, et arrive sur la base de prestations extorquées"(18). M. Weber affirmait en outre en se référant explicitement à la mafia et à la camorra, que "ces prestations sont intermittentes seulement au début lorsqu'elles sont formellement illégales; car ensuite pratiquement, elles prennent souvent le caractère de versements périodiques en échange de prestations déterminées, et spécialement d'une garantie de sécurité"(19).

On peut partir de cette définition pour reconstruire analytiquement les caractères de la mafia. L'élément fondamental est constitué par l'échange qui est fait entre la fourniture d'une garantie de sécurité et le versement d'une somme d'argent ; en second lieu par le fait que la contrepartie monétaire s'établit sur la base d'une extorsion; enfin par la caractéristique selon laquelle l'intermittence du financement prend fin et que ce dernier devient régulier. En reformulant cette interprétation, nous pouvons dire que les mafieux sont des acteurs qui se spécialisent dans l'offre de protection et la fourniture de garanties à des activités et des personnes privées, et que ce rapport tend à devenir stable dans le temps.

Seulement dans la société moderne, la protection des citadins est un monopole exclusif de l'État. Dès lors, s'il existe des personnes privées qui réussissent à assumer avec succès cette fonction, cela signifie que nous sommes en face d'une carence d'efficacité de l'autorité étatique. En outre, par définition, l'offre de la part de citoyens privés d'une prestation qui doit être réservée exclusivement à l'État se situe comme une offre d'un bien illégal. On peut ainsi affirmer que les mafieux sont des gens spécialisés dans l'offre d'un bien illégal, comme la protection privée. Les "cosche" mafieuses sont ainsi des groupes de pouvoir, et plus particulièrement des groupes de pouvoir politique. En tant 
que tels, ils font concurrence à l'État car ils offrent un bien que l'État ne veut, ne peut ou n'est pas en condition d'offrir.

Mais le fond de cette offre réside aussi dans la capacité de ceux qui offrent la protection, de se spécialiser eux-mêmes dans la pratique de la violence. Les deux éléments de la protection et de la violence sont donc étroitement imbriqués, et ce, $\mathrm{d}$ au moins deux manières: d'une part, parce qu'en situation de carence d'efficacité de l'autorité publique, la violence tend à être très diffuse socialement, de l'autre parce que, dans une situation de violence diffuse celui qui veut se substituer à l'autorité étatique comme garant de la protection des biens et de l'intégrité physique des personnes, doit démontrer qu'il a la possibilité de se servir de la force de manière plus efficace que les autres concurrents potentiels. Il doit par conséquent se construire un appareil propre de coercition physique, et à l'égal de l'État, avoir le monopole de la violence sur un territoire déterminé.

Toutefois, à la différence de l'État, qui offre sa protection comme un bien public à tous les citoyens, le mafieux est un "entrepreneur privé de protection" et en tant que tel, il doit faire en sorte que la violence soit toujours présente sur le marché, de façon à ce que la demande de protection ne diminue jamais. Il utilise donc sa spécialité l'exercice de la violence en vue de cette fin pratique. Mettant en action sa capacité, il menace le citoyen privé dans son intégrité physique ou dans ses biens; et en même temps il lui offre de le protéger contre la même menace, en échange de pots de vin: tributs, paiements,... Pour ces raisons, les mafieux sont à la fois des entrepreneurs de la protection et des entrepreneurs de la violence. Or, ce genre d'activité, qui touche à la sphère du pouvoir et de la politique au sens large du terme, c'est-à-dire à l'exercice de la violence comme moyen de domination et d'assujettissement d'un territoire, exige nécessairement une forme d'organisation centralisée. En effet, aucun groupe de pouvoir politique voulant dominer en permanence un territoire, ne peut y réussir sans recourir à des formes d'exaction tributaire stables. Le mécanisme de l'extorsion est justement ce qui garantit son maintien renouvelé dans le temps car dans la mesure où ce pouvoir est socialement reconnu, la domination du territoire est aussi reconnue. $C^{\prime}$ 'est en effet cette domination qui permet de définir les mafieux comme des acteurs qui extorquent aussi l'argent à d'autres criminels, accédant ainsi à une sorte de niveau supérieur de criminalité, à une "métacriminalité". Les besoins de contrôle sur le territoire impliquent d'autre part une centralisation de l'offre de protection et une concentration de la violence, bref, un processus semblable à celui de la formation des États modernes. De là découle le caractère organisé et centralisé de la criminalité mafieuse. Mais cette centralisation ne peut jamais atteindre des dimensions comprenant plusieurs localités parce qu'on courrait le risque d'une rencontre frontale avec l'État, et le risque de devoir se substituer à lui. Or ceci ne rentre pas dans les intérêts des familles, auxquelles un État faible, mais qui existe, est utile, car ses carences sont profitables, d'autant qu'on peut plus facilement le pénétrer.

Nous avons traité jusque là de l'aspect organisé, de la main visible du phénomène mafieux. Mais à côté de cela, il existe également quoiqu'avec des caractères complètement différents, ce qu'un chercheur a appelé "la main invisible ou la criminalité désorganisée"(20). Pour comprendre ce point, il est nécessaire d'insister, non pas sur le fait que les familles ou les groupes mafieux déploient des activités économiques sur des marchés illégaux, mais sur celui que les logiques et les modalités de fonctionnement de ces marchés (comme d'ailleurs celles des marchés légaux) sont 
profondément différentes des logiques et des modalités de fonctionnement du système de la politique et du pouvoir. En effet, sur les marchés illégaux règne le principe de la concurrence qui, sur le plan des fonctions du système de protection-extorsion est réglé par une délimitation rigide des espaces de compétence territoriale. Seulement, à cause de la concurrence, les intérêts qui dérivent des activités économiques sont loin de coïncider avec la loyauté résultant des souverainetés territoriales. Il existe donc structurellement des conflits et des alliances car dans tous les cas de concurrence entre entreprises, on cherche à parvenir à des accords de collaboration et à des alliances pour gouverner le marché. Ainsi, si d'un côté le principe de la souveraineté mafieuse, c'est-àdire l'aspect organisé de la criminalité vaut comme règle très forte même si elle est sujette à violations, il n'empêche que sur le marché illégal, la règle fondamentale n'est pas la monopolisation mais la concurrence, et en conséquence la non-organisation, l'impossibilité de centralisation, bref la main invisible. Ceci est tellement vrai que même Buscetta a confessé aux juges de Palerme que, pour ce qui concerne les affaires, y compris le trafic des stupéfiants, chaque "homme d'honneur" est libre de faire ce qu'il estime être le meilleur(21). L'existence de cette liberté d'initiative économique, de ce droit individuel de poursuivre ses intérêts propres fait alors apparaître, au niveau analytique, une diminution du caractère "organisé" des groupes criminels, au moins en ce qui concerne leurs activités individuelles sur les marchés. Nous devrons donc analyser la criminalité organisée comme une forme de pouvoir qui se manifeste dans l'imposition de pots de vin, dans la pratique de l'extorsion, et qui permet à un groupement politique d'exercer une domination sur un territoire par l'intermédiaire d'un système de prélèvement fiscal.

\section{RÉSUMÉS}

Confrontant les thèses des anthropologues qui ont tendance à voir dans la mafia une structure plurielle; informelle, dispersée sur le territoire, très dépendante de chaque chef de cellule mafieuse (cosche) et se regroupant à des fins pratiques (task oriented), et les thèses des juges palermitains qui ont tendance à considérer la mafia comme une organisation centralisée de type Cosa nostra, l'auteur montre qu'une analyse dynamique permet de dépasser la confrontation: Ainsi en étudiant les capacités d'adaptation de l'organisation et les différents vecteurs qu'elle peut utiliser (violence marché), il déplace les enjeux du débat et repense le phénomène mafieux dans son rapport à l'État en suivant une problématique weberienne.

Confronting, on the one hand, anthropologists theses, with a tendency to see in Mafia a plural and informal structure, spread out territorially, very dependant on local chiefs (cosche) and meeting for task-oriented reasons, to, on the other, the palermitan judges who have a certain tendency to consider Mafia as a Cosa Nostra centralised organisation type, the author shows that a dynamic analysis allows to go further than confrontation. So, by studying the adaptive capacities of the organisation and the different vectors in its use (violence, market), he displaces the debate's stakes, following a weberian problematic, rethinking the Mafia system in its relationship to government. 
INDEX

Index géographique : Europe, Italie, méditerranée

Mots-clés : criminalité, mafias et crime organisé, contrebande et trafic

\section{AUTEUR}

\section{RAIMONDO CATANZARO}

Professeur de sociologie à l'Instituto Carlo Cattaneo, Bologne-Fondation Recherche 